\title{
Directional Differences in the Biaxial Material Properties of Fascia Lata and the Implications for Fascia Function
}

\section{Citation}

Eng, Carolyn M., Francesco Q. Pancheri, Daniel E. Lieberman, Andrew A. Biewener, and Luis Dorfmann. 2014. "Directional Differences in the Biaxial Material Properties of Fascia Lata and the Implications for Fascia Function." Ann Biomed Eng 42 (6) (March 20): 1224-1237. doi:10.1007/s10439-014-0999-3.

\section{Published Version}

doi:10.1007/s10439-014-0999-3

\section{Permanent link}

http://nrs.harvard.edu/urn-3:HUL.InstRepos:22556352

\section{Terms of Use}

This article was downloaded from Harvard University's DASH repository, and is made available under the terms and conditions applicable to Open Access Policy Articles, as set forth at http:// nrs.harvard.edu/urn-3:HUL.InstRepos:dash.current.terms-of-use\#OAP

\section{Share Your Story}

The Harvard community has made this article openly available.

Please share how this access benefits you. Submit a story.

Accessibility 


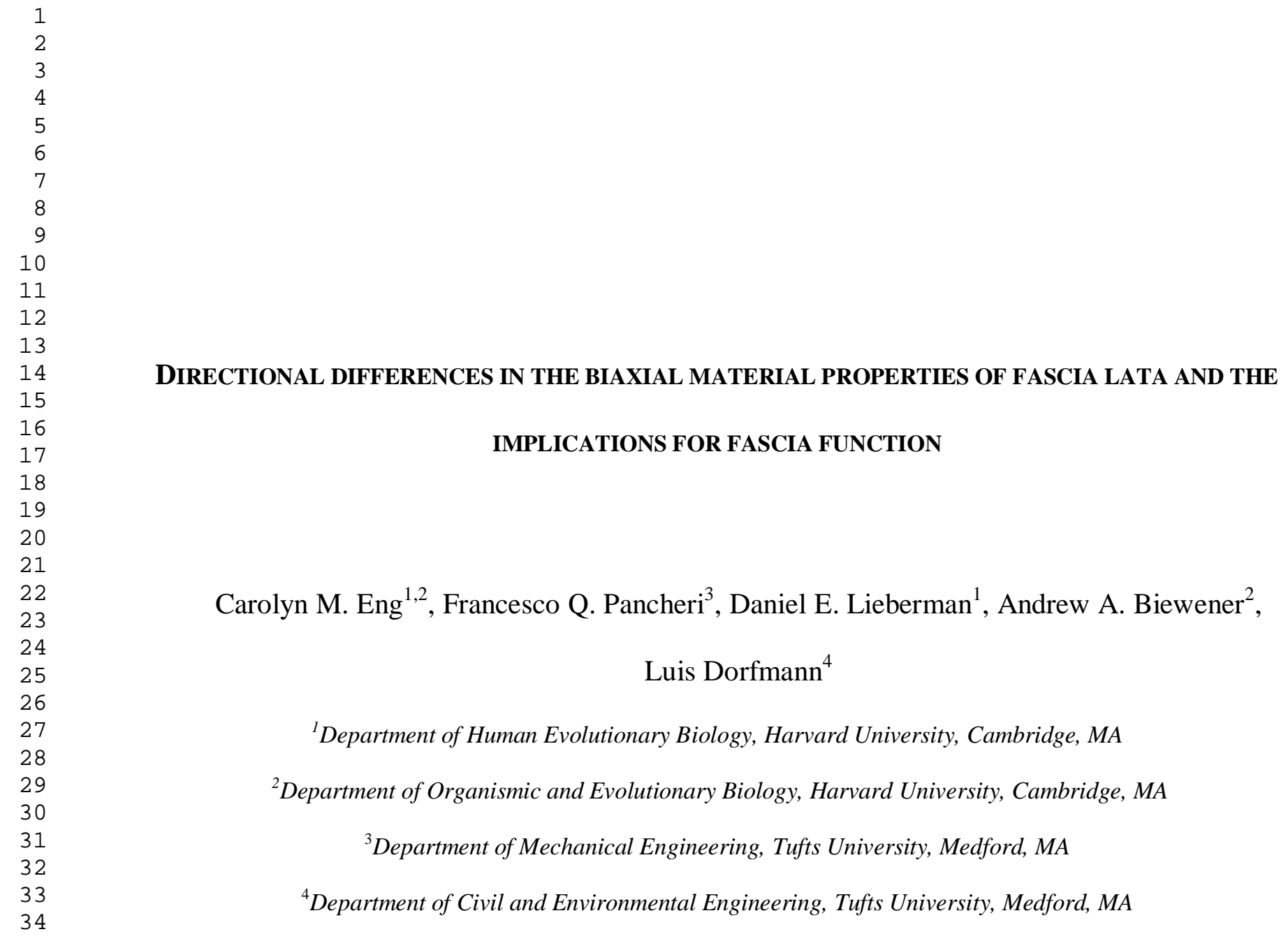

\section{DIRECTIONAL DIFFERENCES IN THE BIAXIAL MATERIAL PROPERTIES OF FASCIA LATA AND THE IMPLICATIONS FOR FASCIA FUNCTION}

\author{
Carolyn M. Eng ${ }^{1,2}$, Francesco Q. Pancheri ${ }^{3}$, Daniel E. Lieberman ${ }^{1}$, Andrew A. Biewener ${ }^{2}$, \\ Luis Dorfmann ${ }^{4}$ \\ ${ }^{1}$ Department of Human Evolutionary Biology, Harvard University, Cambridge, MA \\ ${ }^{2}$ Department of Organismic and Evolutionary Biology, Harvard University, Cambridge, MA \\ ${ }^{3}$ Department of Mechanical Engineering, Tufts University, Medford, MA \\ ${ }^{4}$ Department of Civil and Environmental Engineering, Tufts University, Medford, MA
}

Annals of Biomedical Engineering

Running title: Biaxial material properties of goat fascia lata

Correspondence to: Carolyn M. Eng, Department of Human Evolutionary Biology, 11 Divinity Ave, Cambridge, MA 02138. Phone: (919) 225-0778. E-mail: cmeng@ fas.harvard.edu. Fax: (617) 496-8041. 


\begin{abstract}
Fascia is a highly organized collagenous tissue that is ubiquitous in the body, but whose function remains an enigma. Because fascia has a sheet-like structure attaching to muscles and bones at multiple sites, it is exposed to different states of multi- or biaxial strain. In order to measure how biaxial strain affects fascia material properties, planar biaxial tests with strain control were performed on longitudinal and transversely oriented samples of goat fascia lata (FL). Cruciform samples were cycled to multiple strain levels while the non-cycling perpendicular direction was held at a constant strain. Structural differences among FL layers were examined using histology and SEM. Results show that FL modulus, hysteresis, and strain energy density are greater in the longitudinal versus transverse direction. Increased stiffness in the longitudinal layer is likely due to its greater thickness and greater average fibril diameter compared to the transverse layer(s). Perpendicular strain did not affect FL material properties. Differential loading in the longitudinal versus transverse directions may lead to structural changes, enhancing the ability of the longitudinal FL to transmit force, store energy, or stabilize the limb during locomotion. The relative compliance of the transverse fibers may allow expansion of underlying muscles when they contract.
\end{abstract}

Keywords: Connective tissue, collagen, tissue mechanical properties INTRODUCTION

Fascia is a multilayered collagenous tissue found throughout the body that is in intimate connection with muscles but whose function during movement is not well understood. Various functional roles have been hypothesized for fascia. Because fascia attaches to and envelops many limb muscles, it is often hypothesized that it broadens the insertion of muscles, distributing or redirecting muscle force transmission ${ }^{1-4}$. Several studies have shown that disrupting the fascia 
between or around muscles decreases the muscle's force output ${ }^{1-3}$. Fascial continuity among limb segments and between the limbs and the trunk have led others to posit that fascia plays a role in transferring load and coordinating movement among limb segments and body regions ${ }^{5-7}$. A recent investigation found increased variability in non-sagittal plane movement in the lower limb kinematics of cats after fasciotomy, suggesting that fascia stabilizes the limb by limiting nonsagittal plane movement during locomotion ${ }^{5}$. Finally, an elastic energy storage role has been hypothesized for fascia, wherein energy stored in the fascia during stance phase is recovered to propel the limb forward during swing ${ }^{5,8,9}$.

Fascia's ability to serve these functions depends on its stiffness and resiliency, but these material properties have not been sufficiently characterized. As a multilayered, planar tissue attaching to muscles and bones at multiple sites, fascia is exposed to different states of multiaxial strain. Its functional potential is therefore best captured using biaxial testing protocols, rather than uniaxial tests. The purpose of this study was to measure the biaxial material properties of goat fascia lata (FL) in order to evaluate its potential mechanical functions during movement. We used scanning electron microscopy (SEM) and histology to characterize the structure of the multiple collagen layers in the FL and provide a basis for understanding its material properties.

Data on the relative independence of the multiple collagen layers within fascia is important for understanding its function in vivo. In general, the alignment of collagen fibers differs among layers in fascia ${ }^{10,11}$, a structure which maintains fascial strength when loaded in multiple directions. If the collagen layers are loaded simultaneously in different directions, how does strain in one layer of collagen fibers influence tissue properties in the other layer(s)? Histological studies characterize the collagen layers in fascia as discrete and separated by layers of loose connective tissue, a structural organization which is thought to allow the collagen layers 
to move independently ${ }^{10}$. However, studies describing the biaxial material properties of other biological tissues including skin, aponeurosis, tendon, and aortic tissue have shown that tissue properties vary substantially when strained uniaxially versus biaxially. For instance, tissue stiffness is greater in biaxial versus uniaxial tests ${ }^{12,13}$ and stiffness in the collagen fiber direction increases with strain in the orthogonal direction ${ }^{14,15}$. Whether the same is true for fascia has not been investigated. The potential for biaxial strain to modulate fascia stiffness is particularly important for a tissue that may play such diverse functional roles. For example, during a movement, fascia's stiffness may be modulated depending on its role in limb stability versus energy storage. Planar biaxial testing with strain control provides an opportunity to investigate if fascia stiffness can be modulated by different biaxial strain conditions.

Finally, data on the relative independence of collagen layers in fascia are important for creating musculoskeletal models that are sufficiently accurate to explain fascia function. In commonly used musculoskeletal models (e.g., Arnold et al., 2010), muscles are represented as independent muscle-tendon units that attach to bones at discrete points proximally and distally. These models do not consider the three-dimensional strain environment that may occur in tissues when multiple muscles insert in a single connective tissue, when muscles attach to connective tissues both proximally and anteriorly or posteriorly, or when forces are transmitted both longitudinally and transversely. Furthermore, connective tissues in these models are often assumed to have the same stiffness as tendon, which may be inaccurate for fascia. Data on the effect of biaxial strains on fascia material properties will indicate whether the 3D strain environment of planar connective tissues can be accurately characterized using discrete and independent muscle-connective tissue units. 
Here we focus on the fascia lata, which is found on the lateral side of the thigh of mammals. In goats, the gluteobiceps (GB) and tensor fascia lata (TFL) muscles insert into the FL. These muscles are knee extensors and antagonists at the hip joint (GB extends and TFL flexes the hip). The goat FL is composed of two primary orientations of highly aligned collagen fibers (assemblies of collagen fibrils and considered here to be synonymous with collagen fascicles), which are visible both microscopically and macroscopically (Figure 1). The collagen fibers in the longitudinal layer(s) run approximately parallel with the femur and are parallel with the muscle fibers of TFL, while the collagen fibers in the transverse layer(s) are at a $67-80^{\circ}$ angle to the longitudinal fibers ${ }^{17}$ and are parallel with the inserting GB muscle fibers. Our histological analysis of the FL will reveal whether there is more than one layer of tissue within each primary collagen orientation. The FL is thickened relative to other limb fascia, suggesting that it has the potential to play a role in limb stability, force transmission, and/or energy storage. As with other fascia, however, the function of the FL during movement has not been well explored.

Based on the anatomy and hypothesized function of the FL and biaxial behavior of other connective tissues, we propose five hypotheses relating the biaxial material behavior of the goat FL to its functional role during movement. Although the attachments and inserting muscles load the fascia lata biaxially, goat locomotor kinematics occur primarily in the sagittal plane, likely creating larger longitudinal than transverse loads in the tissue. We therefore hypothesize that larger longitudinal loads necessitate greater longitudinal elastic modulus (H1). We hypothesize that perpendicular strain increases the elastic modulus of the FL in its primary, longitudinal loading axis (H2), consistent with prior studies of other connective tissues. We hypothesize that increased perpendicular strain preloads the FL, decreasing the length of the toe and heel region of the stress-strain curve (H3). We also hypothesize that the increase in tissue modulus with 
perpendicular strain increases the strain energy stored in the FL at a given strain (H4). Finally, we hypothesize that differences in material properties between longitudinal and transverse samples are due to differences in collagen layer thickness and collagen fibril size (H5).

\section{MATERIALS AND METHODS}

FL samples were obtained from hindlimbs of five adult goats (mean \pm SD: $35.1 \pm 7.3 \mathrm{~kg}$ ) from Blood Farm (Groton, MA). The FL was dissected from the hindlimb within two hours and all tests were performed within 48 hours of sacrifice. The FL was removed from the skinned hindlimb, wrapped in saline-soaked gauze, and stored at $4{ }^{\circ} \mathrm{C}$ prior to testing and between tests. Two samples were cut from each FL, one oriented parallel to the longitudinal fibers while the other was oriented parallel to the transverse fibers (Figure 2). Samples were cut using a custommade cruciform-shaped die with a central gage region measuring $12 \mathrm{~mm}$ x $12 \mathrm{~mm}$. The die was aligned visually with the fiber orientation of one family of fibers (i.e., longitudinal or transverse fibers) and cut using pressure evenly distributed over the die. Because the two families of fibers are not orthogonal, the perpendicular arms of the die could not be aligned with both fiber directions in a single sample. Sample thickness was measured at several points in the central gage region and averaged across regions using a dial thickness gage accurate to $0.01 \mathrm{~mm}$ (Mitutoyo, Sakato, Japan). Gage marks were arranged to correspond to each of the four arms of the cruciform sample (Figure 3) and were attached to the gage region using biocompatible glue (Loctite, Westlake, OH). A distance of 7-10 mm separated opposing pairs of gage marks. Each arm was secured in custom-made extension platens covered in fine grit sand paper (Figure 3A). Biaxial testing

Biaxial tests with strain control were performed using a custom built Zwick/Roell planar biaxial testing system (Ulm, Germany) ${ }^{18}$. The sample was attached to the four linear 
independently controlled actuators via rigid pincer type grips (Figure 3B). The tissue was maintained at $39^{\circ} \mathrm{C}$ during testing via a temperature-controlled chamber filled with phosphate buffered saline (PBS). All tests were performed using simultaneous non-contact strain controlled measurements using a video extensometer rigidly fixed to the machine's frame. Images of the sample were recorded prior to testing to measure specimen width in the gage region, which was subsequently used with the thickness measure to calculate sample cross-sectional area.

In the testing procedure, the perpendicular strain $\left(\varepsilon_{\mathrm{P}}\right.$; strain exerted on the tissue arms perpendicular to the arms aligned with one fiber family) was set at a pre-determined strain of $0 \%$ or $3 \%$ and held constant while the tissue was cycled five times each to cycling strains $\left(\varepsilon_{\mathrm{C}}\right)$ of $2 \%$, $4 \%, 6 \%$, and $8 \%$. The tissue was returned to the initial length in the cycling direction $\left(\mathrm{L}_{0, \mathrm{C}}\right)$ at the end of each cycle. A 180-second rest period was applied after each cycling strain. The testing protocol is summarized in Table 1 and was the same for both longitudinally and transverselyoriented samples. At each cycling strain level, $\varepsilon_{\mathrm{P}}$ was first set to $0 \%$ while $\varepsilon_{\mathrm{C}}$ was cycled and then $3 \%$ while $\varepsilon_{C}$ was cycled again to the same strain. The sample's initial reference configuration was established as the length in the cycling and perpendicular directions when a nominal load of $80 \mathrm{mN}$ was reached in each direction. Prior to testing, the sample was pre-conditioned by performing the described protocol to $\varepsilon_{\mathrm{C}}$ of $2 \%$ while holding $\varepsilon_{\mathrm{P}}$ at $0 \%$ then $3 \%$. The sample reference configuration was re-determined after pre-conditioning. All tests were performed at a strain rate of $0.15 \% / \mathrm{s}$ and recorded at $12.5 \mathrm{~Hz}$. Data were expressed as engineering stress and strain, which were calculated using the sample cross-sectional area and $\mathrm{L}_{0, \mathrm{C}}$, respectively.

The stress-strain curves for each sample were used to calculate initial stress, maximum stress, initial modulus, transition strain, elastic modulus, strain energy density, and hysteresis. These parameters were calculated and averaged over cycles $2-5$ at a strain of $6 \%$. A strain of $6 \%$ 
was chosen for data analysis because it included both the nonlinear and linear portions of the stress-strain curve and damage often occurred at the greater cycling strain of $8 \%$. Initial stress was determined as the stress in the cycling direction at a strain of $0 \%$ after the perpendicular strain was set at $0 \%$ or $3 \%$. We expect initial stress to be greater than zero because the reference configuration was set at a preload of $80 \mathrm{mN}$. Maximum stress was determined as the stress at $6 \%$ strain. Initial modulus was calculated by determining the derivative of a linear polynomial fit to the stress-strain curve from a cycling strain of $0 \%$ to $1.5 \%$. To find the transition point where the stress-strain curve became linear, a linear polynomial equation was incrementally fit to the stress-strain curve for successive increments of $1.5 \%$ cycling strain using a least-squares curve fit. The transition point was determined when the $\mathrm{R}^{2}$ of the fit was greater than 0.997 and the slope of the linear fit was greater than $70 \%$ of the maximum slope for that stress-strain curve. The initial strain value at the first point of this increment was determined as the transition strain. The slope of the linear polynomial after the transition point was calculated as the sample's elastic modulus. Strain energy density was calculated as the integral of the loading stress-strain curve. Hysteresis was calculated as the percent difference in loading and unloading work, which were calculated as the integrals of the loading and unloading stress-strain curves, respectively.

\section{Fascia structure}

After removing samples for testing, four $1 \mathrm{~cm}$ x $1 \mathrm{~cm}$ samples were cut from the remaining fascia. For each of the two imaging techniques (histology and SEM), one sample was cut normal to the longitudinal fibers while a second sample was cut normal to the transverse fibers. All samples were obtained from the mid-region of each fascia lata, pinned to polystyrene, and covered with fine plastic mesh to keep the sample flat during fixation. 
Samples used for SEM were fixed in $4 \%$ paraformaldehyde overnight, washed with $1 \mathrm{x}$ PBS, and serially dehydrated to $100 \%$ ethanol. Samples were critical point dried (Tousimis Autosamdri-815, Rockville, MD), mounted on an aluminum stub, and sputter coated with platinum-palladium (Cressington 208HR, Watford, UK). Imaging was performed using a field emission scanning electron microscope (Zeiss Supra55VP, Jena, Germany) at an accelerating voltage of $3.0 \mathrm{kV}$. The diameter of collagen fibrils was measured using ImageJ (NIH, Bethesda, MD).

Samples used for histological sectioning and staining were fixed in neutral-buffered formalin overnight, washed with 1x PBS, dehydrated, cleared with xylene, and infiltrated with Paraplast (Structure Probe Inc., West Chester, PA). Samples were oriented perpendicular to the face of the embedding block and embedded in Paraplast. All paraffin-embedding procedures were performed using a Tissue-Tek VIP tissue processor (MilesCo Scientific, Princeton, MN). From the paraffin-embedded samples, 6-8 $\mu \mathrm{m}$ sections were obtained and stained using Mallory’s trichrome. Samples were imaged with a Leica DMR microscope (Solms, Germany) equipped with a QImaging Retiga 2000r camera (Surrey, Canada). The thickness of the collagen layer(s) normal to the section plane was measured using ImageJ and averaged across sections.

\section{Statistics}

A multivariate linear mixed model fit by maximum likelihood was used to examine the effect of perpendicular strain on tissue properties including initial stress, initial modulus, transition strain, elastic modulus, strain energy, and dissipation and to compare properties between the longitudinal and transverse tissue samples. This model accounts for repeated measures made on non-independent parameters and was implemented using R statistical 
software $^{19}$. Prior to fitting the model, parameters were transformed to a common log scale and then transformed to z-scores.

In the model, fixed effects included fascia orientation, perpendicular strain, and the parameters calculated from the stress-strain curve, and the random effects included random intercepts for cycle number nested within subject. The model included a first order autoregressive moving average correlation structure for the residuals to account for the correlation between the parameters and cycle number. Log-transformed residuals were examined to validate the model's assumption of normality and planned pair-wise comparisons were made using the model's contrast matrix with a sequential Bonferroni correction to account for multiple comparisons $^{20}$. Structural measures were compared between orientations using a paired samples t-test. All results were considered significant at a level of $\mathrm{p}<0.05$ and are reported as mean \pm standard error of sample mean (s.e.m.).

\section{RESULTS}

Representative stress-strain curves for longitudinally and transversely oriented samples in Figure 4 show behavior characteristic of collagenous tissues with toe, heel, and linear regions of increasing stiffness. The tissue also demonstrated moderate hysteresis in both orientations. Lower stress in the tissue for a given strain in the transverse orientation compared to the longitudinal orientation indicates greater compliance in the transverse versus longitudinal orientation. Substantial decreases in stress with subsequent cycles at $8 \%$ strain in both orientations likely indicate more extensive tissue damage at this strain level (Figure 4).

The effect of sample orientation (longitudinal versus transverse) had a greater influence on material properties than perpendicular strain $(0 \%$ versus $3 \%)$, as demonstrated by the multivariate linear mixed model illustrated in Figure 5. The two-way interaction between 
orientation and perpendicular strain was not significant and a multivariate model that included this interaction term was not a significantly better fit than a simpler, more parsimonious model that excluded it. Therefore, the latter model was chosen to analyze these data. The nonsignificant interaction between orientation and perpendicular strain indicates that the effect of perpendicular strain does not depend on whether the sample was longitudinal or transversely oriented. Note that while we present means for comparison across orientation and perpendicular strain, the statistical model compared orientation and perpendicular strain within individual animals and lumped these comparisons across animals.

\section{Effect of sample orientation on material properties}

Between longitudinal and transverse orientations there was a significant difference in most variables describing the shape of the stress-strain curve except transition strain (Table 2). Initial modulus was significantly greater in the longitudinal versus transverse orientation $(45.8 \pm$ 14.8 MPa vs. $12.1 \pm 3.5 \mathrm{MPa}$; $\mathrm{p}<0.001)$. Initial stress and maximum stress were also significantly greater in the longitudinal versus transverse orientation (Initial stress: $307.7 \pm 206.5$ kPa vs. $75.6 \pm 61.6 \mathrm{kPa}$; $\mathrm{p}<0.05$ and maximum stress: $9357.1 \pm 1106.3 \mathrm{kPa}$ vs. $1947.5 \pm 254.0$ $\mathrm{kPa} ; \mathrm{p}<0.001)$. The transition strain was $3.3 \pm 0.3 \%$ in the longitudinal orientation and $3.1 \pm 0.3 \%$ in the transverse orientation and was not significantly different between orientations. Elastic modulus was significantly greater in the longitudinal versus transverse orientation $(263.5 \pm 16.3$ MPa vs. $52.2 \pm 4.7 \mathrm{MPa} ; \mathrm{p}<0.001)$. Across individuals, longitudinally oriented samples had on average a 4.4 times greater elastic modulus than transverse samples. Strain energy density was also significantly greater in the longitudinal than transverse orientation $\left(197.2 \pm 38.2 \mathrm{~mJ} / \mathrm{m}^{3}\right.$ vs. $\left.39.7 \pm 9.1 \mathrm{~mJ} / \mathrm{m}^{3} ; \mathrm{p}<0.001\right)$. Hysteresis was also significantly greater in the longitudinal than transverse orientation $(14.8 \pm 1.6 \%$ vs. $10.1 \pm 1.3 \% ; \mathrm{p}<0.001)$. 


\section{Effect of perpendicular strain on material properties}

Although tissue orientation had a large effect on FL material properties, an increase in perpendicular strain did not significantly alter the stress-strain curve in either longitudinal or transverse orientations (Figure 6; Table 3). The parameters describing the shape of the stressstrain curve including initial modulus, transition strain, and elastic modulus did not significantly change when perpendicular strain was increased from $0 \%$ to $3 \%$ ( $p>0.05$ ). Perpendicular strain also did not have a significant effect on hysteresis or strain energy density. While initial stress significantly increased as perpendicular strain increased from $0 \%$ to $3 \%$ in the longitudinal $(104.5 \pm 118.1 \mathrm{kPa}$ vs. $511.0 \pm 396.5 \mathrm{kPa} ; \mathrm{p}<0.001)$ and transverse orientations $(11.2 \pm 7.2 \mathrm{kPa}$ vs. $140.0 \pm 122.3 \mathrm{kPa} ; \mathrm{p}<0.001)$, maximum stress did not significantly change $(\mathrm{p}>0.05)$.

\section{Fascia structure}

The average total thickness of fascia lata samples was $0.32 \pm 0.02 \mathrm{~mm}$. As figure 7 illustrates, histology revealed that there was only one layer of longitudinal collagen fibers, while many fascia samples had two layers of transverse collagen fibers. The thickness of the longitudinal layer of collagen fibers was significantly greater than the transverse layer(s) (218.0 \pm 22.7 vs. $94.7 \pm 6.5 \mu \mathrm{m} ; \mathrm{p}<0.05)$. Across samples, the average ratio of longitudinal to transverse layer thickness was $2.4 \pm 0.3$ (Figure 7; Table 4). The collagen fibrils in the longitudinal layer were significantly larger compared to those in the transverse layer(s) (Figure 7; Table 4). The average diameter of the longitudinal fibrils was $127.0 \pm 9.2 \mathrm{~nm}$, while the average diameter of the transverse fibrils was $94.9 \pm 8.9 \mathrm{~nm}(\mathrm{p}<0.05)$.

\section{DISCUSSION}

Our results demonstrate that goat FL orientation significantly affects tissue material properties including initial and maximum stress, initial modulus, elastic modulus, hysteresis, and 
strain energy density. On the other hand, counter to several of our hypotheses (H2, H3, \& H4), an increase in the constantly held perpendicular strain caused a significant increase in initial stress but did not significantly alter any other material properties measured in this study. We show that the differences in material properties between the two collagen orientations are related to structural differences between the longitudinal and transverse collagen layers.

\section{Effect of perpendicular strain and sample orientation on material properties}

We found that an increase in perpendicular strain from $0 \%$ to $3 \%$ did not greatly alter the biaxial material properties of FL. Initial modulus, hysteresis, and maximum stress did not significantly change with an increase in perpendicular strain. Contrary to Hypothesis 2, elastic modulus did not increase with increased perpendicular strain, and strain energy density also did not increase with perpendicular strain as hypothesized (H4). We hypothesized that increased perpendicular strain would preload the tissue and decrease transition strain in the cycling direction (H3), yet transition strain did not change with perpendicular strain. However preloading from perpendicular strain did cause a significant increase in initial stress. Because deformation of biological tissues is isovolumetric ${ }^{21}$, when the FL was strained perpendicularly prior to cycling, the tissue in the cycling direction must contract. Since our protocol maintained the tissue in the cycling direction at a constant strain as initial deformation occurred in the perpendicular direction, initial stress must increase. Interestingly, although this was the case (Table 3), maximum stress did not significantly increase, indicating that perpendicular strain does not merely cause the stress-strain curve to shift upwards leading to higher stresses at all strain values. While there was little effect of perpendicular strain on tissue properties, a large influence of tissue orientation on biaxial material behavior was observed. Compared to the transversely oriented tissue, the tissue samples cut and cycled in the longitudinal direction had significantly 
greater initial stress, initial modulus, maximum stress, hysteresis, and strain energy density

(Figure 6). Transition strain did not differ between orientations. As hypothesized, elastic modulus was significantly greater in the longitudinal direction $(\mathrm{H} 1)$.

\section{Structural differences and their influence on material properties}

Differences in material properties between the longitudinal and transverse orientations can largely be explained by differences in tissue structure as hypothesized (H5). The more than two-fold greater thickness in the longitudinal layer compared to the transverse layer(s) likely played a role in the longitudinal samples' greater initial and elastic modulus. Histological sections revealed that two transverse layers often flanked a single longitudinal layer. Cross-links within and between collagen molecules are important for determining the stiffness of collage ${ }^{22}$,

23. The discontinuity between transverse layers and subsequent increase in surface area to volume ratio in the transverse layer(s) likely decreases the number of cross-links forming in the transverse layer, which may contribute to the decreased transverse elastic modulus we observed. Because the discrepancy in longitudinal versus transverse layer thickness is only detectable using microscopy, the sample cross-sectional areas used to estimate tissue stress did not take this difference into account. Therefore the longitudinal and transverse layers of fascia lata are not mechanically equivalent and our stress calculations likely overestimate the actual load-bearing component of the tissue, especially in the transverse orientation. Nevertheless, although we may underestimate the intrinsic strength of the collagen in the transverse layer(s), our calculations capture the functionally relevant characteristics of the fascia's biaxial response as it would respond in vivo. Under a given muscle load transmitted to the FL, the FL will undergo larger strains in its transverse versus longitudinal orientation because of structural 
present a material model of the FL that accounts for its multilayered structure.

Differences in material properties with respect to fascia orientation are also likely due to differences in collagen fibril size. Electron microscopy results showed that the collagen fibrils in the longitudinal layer have a larger average diameter than the fibrils in the transverse layer(s). Previous research has shown that collagen fibril size correlates with increased tensile strength and elastic modulus in connective tissues ${ }^{24,25}$. Together, these structural differences between the longitudinal and transverse orientations explain the tissue's greater elastic modulus, initial and maximum stress, and strain energy density in the longitudinal versus transverse orientation.

Finally, collagen fiber alignment is another factor that potentially contributes to the greater initial modulus in longitudinal versus transverse fascial orientations. More highly aligned fibers in the longitudinal orientation could explain the increased initial modulus in the longitudinally oriented samples. Tissues with more aligned collagen fibrils are stiffer, and imaging of tissue during mechanical testing shows that fibers re-orient in the direction of loading under tension $^{26-29}$. Fiber re-alignment in the transverse orientation may also lead to increased hysteresis. Hysteresis would also be expected to be greater in the transverse orientation due to smaller fibril size with higher surface area-to-volume ratio, increasing shear stresses that result from interactions between the fibrils and the matrix components ${ }^{22,25}$. However, greater hysteresis was observed in the longitudinal direction. A possible explanation for this result is that hysteresis was compared for cycles 2-5, but re-alignment may have occurred in the first cycle. Consistent with this, when cycling to $6 \%$ strain, hysteresis was $131 \%$ higher in the first cycle relative to subsequent cycles in the transversely oriented samples, while hysteresis is only $30 \%$ higher in the first cycle for samples cycled in the longitudinal direction. The greater energy loss in the first 
cycle relative to subsequent cycles in the transverse samples indicates that the FL's transverse layer(s) have lower collagen fiber alignment causing substantial realignment during testing.

\section{Comparisons with other studies}

Contrary to previous findings that an increase in perpendicular strain causes an increase in elastic modulus in other connective tissues including aponeurosis ${ }^{14}$ and shark skin ${ }^{15}$, our results demonstrate that the elastic modulus of goat FL does not change with increased perpendicular strain. One reason for the discrepancy in our results may be that higher perpendicular strains of up to $10 \%$ were reached in the aponeurosis, while we imposed a maximum perpendicular strain of 3\%. Additionally, Wainwright and co-authors may have imposed higher perpendicular strains on the shark skin than the 3\% strain used here but their study only reports perpendicular stresses.

The material properties of fascia described here are relatively consistent with results from other studies describing material properties of connective tissues including fascia, tendon, and aponeurosis (Table 5). Our measure of longitudinal stiffness $(263.5 \pm 16.3 \mathrm{MPa})$ is lower than measures of fascia lata stiffness from dogs $(390 \mathrm{MPa})^{8}$ and humans $(397.5 \pm 17.1 \mathrm{MPa})^{30}$. One reason for the difference compared with dog fascia is that Bennett et al. (1989) described peak elastic modulus whereas the modulus reported here is was based on the initial linear portion of the stress-strain curve and peak modulus values can be higher. Additionally, Bennett et al. measured strain using grip-to-grip separation, while in our study and in Butler et al. (1984), true strain was measured by tracking fiducial markers in the sample gage region. The lower stiffness values in our study may also be due to interspecific differences in tissue properties.

Our stiffness values for the longitudinal and transverse FL are also lower than measures of longitudinal and transverse stiffness in turkey aponeurosis ${ }^{31}$. The lower transverse FL stiffness 
compared to aponeurosis is particularly puzzling because our FL tests were performed in alignment with a layer of collagen fibers in the transversely oriented FL and these collagen fibers are absent from aponeurosis. While our results show that the goat FL is less stiff than tendon, which has elastic modulus values ranging from $300-2000 \mathrm{MPa}^{30,32,33}$, hysteresis in FL and tendon is similar, suggesting that fascia has similar resiliency compared to tendon (Table 5).

\section{Fascia function}

The biaxial properties of FL measured here have implications for inferring its in vivo function. In order to play an appreciable role in elastic energy storage, force transmission, or limb stability, the fascia must be relatively stiff when loaded by muscle or stretched by joint motion. Because the GB and TFL muscle fibers insert orthogonally in the FL, nearly orthogonal collagen fiber families allow the FL to transmit loads from both muscles. The increased tissue stiffness achieved by reinforcement with the transverse fibers may play another role in fascia function. Studies exploring the role of fascia in mediating muscle force output show that fascia may play a role in maintaining intramuscular pressure ${ }^{1,4}$. However, fascia often covers or envelops muscles, and thus must be sufficiently compliant to allow underlying muscles to expand during contraction. By expanding circumferentially, muscles will stretch the fascia transversely. The relatively low transverse stiffness observed here would allow this expansion. The transverse layer of FL fibers may provide adequate tissue stiffness to transmit muscle forces and maintain intramuscular pressure, while their relative compliance would facilitate expansion of underlying muscles during contractions.

To sum up, the data presented here provide an important first step in understanding the function of the myriad planar collagenous tissues found throughout the body. Unexpectedly, our results indicate that fascia material properties are not strongly influenced by their biaxial strain 

than in vivo loading rates. Previous studies, however, have shown that strain rate has little effect on the elastic modulus of other connective tissues ${ }^{34}$. The maximum perpendicular strain of $3 \%$ used here may be too low to see a significant effect on FL material properties. However, when we used higher perpendicular strains, the FL samples failed before reaching cycling strains of $6 \%$. Additionally, the testing protocol used here was performed with a non-randomized order of strains increasing from cyclic strains of $2 \%$ to $8 \%$, with perpendicular strains of $0 \%$ and subsequently $3 \%$ strain in order to avoid causing tissue damage. Even so, we did observe evidence of damage in the tissue at or above $3 \%$ perpendicular strain. Longitudinal and transverse tests both exhibited evidence of stress softening at increasing strains (Figure 4), indicating disruption of matrix components leading to a loss of stiffness and increased 
if the collagen fibers in the transverse orientation did not always extend from grip to grip.

To summarize, a strain controlled planar biaxial protocol was applied to goat FL samples. These results demonstrate that although the collagen layers in the goat FL are not entirely independent, they do not appear to strongly influence one another's material properties when loaded in opposing directions during biaxial testing. Directional differences in tissue properties are likely due to structural differences between transverse versus longitudinal layer(s) of the FL including layer thickness and collagen fibril size. The differing material properties observed between the longitudinal and transverse orientations suggest that differential loading in these directions during growth and development may lead to these structural changes, enhancing the ability of the longitudinal FL to transmit force, store energy, and/or stabilize the goat limb. These data are important for generating more accurate musculoskeletal models of fascia function.

\section{ACKNOWLEDGEMENTS}

The authors gratefully acknowledge Richard Blood at Blood Farm for help in procuring goat hindlimbs. We are grateful to Steven Worthington and Simo Goshev of the research consulting team at Harvard's Institute for Quantitative Social Sciences for their assistance with statistical analysis. We thank Zachary Lewis and James Weaver for their help with tissue imaging techniques. This work was performed in part at the Harvard University Center for Nanoscale Systems, which is supported by the National Science Foundation under award no. ECS-0335765. 


\section{References}

1. Garfin S. R., C. M. Tipton, S. J. Mubarak, S. L. Woo, A. R. Hargens, W. H. Akeson. Role of fascia in maintenance of muscle tension and pressure. J Appl Physiol. 51(2):317320,1981 .

2. Huijing P. A., G. C. Baan. Myofascial force transmission causes interaction between adjacent muscles and connective tissue: effects of blunt dissection and compartmental fasciotomy on length force characteristics of rat extensor digitorum longus muscle. Arch Physiol Biochem. 109(2):97-109,2001.

3. Huijing P. A., H. Maas, G. C. Baan. Compartmental fasciotomy and isolating a muscle from neighboring muscles interfere with myofascial force transmission within the rat anterior crural compartment. J Morphol. 256(3):306-321,2003.

4. Mozan L. C., R. D. Keagy. Muscle relationships in functional fascia: A preliminary study. Clin Orthop. 67:225,1969.

5. Stahl V. A. A biomechanical analysis of the the role of the crural fascia in the cat hindlimb: Georgia Institute of Technology and Emory University; 2010.

6. Dubiel W. T., A. Wigren. Functional Status of the Lower Extremity After Resection of Fascia Lata: A Clinical and Physiological Follow-up Study in Patients with Fascia Lata Heart Valve Replacement. Acta Orthopaedica. 45(1-4):599-613,1974.

7. Barker P. J., C. A. Briggs, G. Bogeski. Tensile transmission across the lumbar fasciae in unembalmed cadavers: effects of tension to various muscular attachments. Spine. 29(2):129138,2004 .

8. Bennett M. B. A possible energy-saving role for the major fascia of the thigh in running quadrupedal mammals. J Zool. 219:221-230,1989. 
9. Eng C. M., D. E. Lieberman, A. A. Biewener. In vivo strain patterns indicate different functions in the proximal and distal fascia lata of the goat. Society for Integrative and Comparative Biology Annual Meeting; Charleston, S.C.2012.

10. Stecco C., V. Macchi, A. Porzionato, F. Duparc, R. De Caro. The fascia: the forgotten structure. Ital J Anat Embryol. 116(3):127-138,2011.

11. Benjamin M. The fascia of the limbs and back--a review. J Anat. 214:1-18,2009.

12. Lanir Y., Y. Fung. Two-dimensional mechanical properties of rabbit skin-II. Experimental results. J Biomech. 7(2):171-182,1974.

13. Vande Geest J. P., M. S. Sacks, D. A. Vorp. The effects of aneurysm on the biaxial mechanical behavior of human abdominal aorta. J Biomech. 39(7):1324-1334,2006.

14. Azizi E., T. J. Roberts. Biaxial strain and variable stiffness in aponeuroses. The Journal of Physiology. 587(17):4309,2009.

15. Wainwright S. A., F. Vosburgh, J. H. Hebrank. Shark skin: function in locomotion. Science. 202(4369):747-749,1978.

16. Arnold E. M., S. R. Ward, R. L. Lieber, S. L. Delp. A model of the lower limb for analysis of human movement. Ann Biomed Eng. 38(2):269-279,2010.

17. Pancheri F. Q., C. M. Eng, D. E. Lieberman, A. A. Biewener, L. Dorfmann. A constitutive description of the anisotropic response of the fascia lata. Journal of the Mechanical Behavior of Biomedical Materials. 30:306-323,2014.

18. Pancheri F. Q., A. L. Dorfmann. Strain controlled biaxial tension of natural rubber: New experimental data. Rubber Chemistry and Technology. , In press,2013.

19. R Core Team. R: A language and environment for statistical computing. Vienna, Austria: R Foundation for Statistical Computing; 2012. 
20. Holm S. A simple sequentially rejective multiple test procedure. Scandinavian Journal of Statistics. 6:65-70,1979.

21. Qiu G., T. Pence. Remarks on the behavior of simple directionally reinforced incompressible nonlinearly elastic solids. Journal of Elasticity. 49(1):1-30,1997.

22. Puxkandl R., I. Zizak, O. Paris, et al. Viscoelastic properties of collagen: synchrotron radiation investigations and structural model. Philos Trans R Soc Lond B Biol Sci. 357(1418):191-197,2002.

23. Davison P. F. The contribution of labile crosslinks to the tensile behavior of tendons. Connect Tissue Res. 18(4):293-305,1989.

24. Robinson P. S., T. W. Lin, A. F. Jawad, R. V. Iozzo, L. J. Soslowsky. Investigating tendon fascicle structure-function relationships in a transgenic-age mouse model using multiple regression models. Ann Biomed Eng. 32(7):924-931,2004.

25. Parry D. A. D. The molecular and fibrillar structure of collagen and its relationship to the mechanical properties of connective tissue. Biophys Chem. 29:195-209,1988.

26. Lake S. P., K. S. Miller, D. M. Elliott, L. J. Soslowsky. Effect of fiber distribution and realignment on the nonlinear and inhomogeneous mechanical properties of human supraspinatus tendon under longitudinal tensile loading. J Orthop Res. 27(12):1596-1602,2009.

27. Szczesny S. E., J. M. Peloquin, D. H. Cortes, J. A. Kadlowec, L. J. Soslowsky, D. M. Elliott. Biaxial tensile testing and constitutive modeling of human supraspinatus tendon. J Biomed Eng. 134:021004,2012.

28. Sellaro T. L., D. Hildebrand, Q. Lu, N. Vyavahare, M. Scott, M. S. Sacks. Effects of collagen fiber orientation on the response of biologically derived soft tissue biomaterials to cyclic loading. Journal of Biomedical Materials Research Part A. 80(1):194-205,2007. 
29. Tower T. T., M. R. Neidert, R. T. Tranquillo. Fiber alignment imaging during mechanical testing of soft tissues. Ann Biomed Eng. 30(10):1221-1233,2002.

30. Butler D. L., E. S. Grood, F. R. Noyes, R. F. Zernicke, K. Brackett. Effects of structure and strain measurement technique on the material properties of young human tendons and fascia. J Biomech. 17(8):579-596,1984.

31. Azizi E., G. M. Halenda, T. J. Roberts. Mechanical properties of the gastrocnemius aponeurosis in wild turkeys. Integrative and Comparative Biology. 49(1):51-58,2009.

32. Pollock C. M., R. E. Shadwick. Allometry of muscle, tendon, and elastic energy storage capacity in mammals. Am J Physiol. 266:R1022-1031,1994.

33. Bennett M. B., R. F. Ker, N. J. Dimery, R. M. Alexander. Mechanical properties of various mammalian tendons. J Zool. 209(4):537-548,1986.

34. Ker R. F. Dynamic tensile properties of the plantaris tendon of sheep (Ovis aries). J Exp Biol. 93:283-302,1981.

35. Peña E. Prediction of the softening and damage effects with permanent set in fibrous biological materials. Journal of the Mechanics and Physics of Solids. 59(9):1808-1822,2011. 36. Dorfmann A. L., B. A. Trimmer, W. A. Woods. A constitutive model for muscle properties in a soft bodied arthropod. Journal of the Royal Society Interface. 4:257-269,2007. 


\section{Figure Legends}

Figure 1. The transverse and longitudinal collagen layers in the fascia lata are each composed of highly aligned collagen fibers that are visible without (A) and with magnification (B). Gray and black lines in (A) represent the orientation of the transverse and longitudinal collagen fiber families, respectively. The sample in (B) was dehydrated in methanol, cleared in Benzyl Alcohol: Benzyl Benzoate (3:1) and imaged using differential interference contrast microscopy. Scale bar is $40 \mathrm{~mm}$ in (A) and $0.5 \mathrm{~mm}$ in (B).

Figure 2. Longitudinal $\left(\mathrm{FL}_{\mathrm{L}}\right)$ and transverse $\left(\mathrm{FL}_{\mathrm{T}}\right)$ samples were cut from the fascia lata of each goat using a custom cruciform die (inset).

Figure 3. Gage marks were attached to each arm of the cruciform fascia lata sample and samples were mounted in extension platens with fine grit sand paper (A). Tests were performed in a Zwick/Roell biaxial testing system (B), which consisted of four independent actuators as described in Pancheri and Dorfmann ${ }^{18}$.

Figure 4. Representative data from longitudinally (A) and transversely (B) oriented fascia lata samples. Data shown are from cycles $2-5$ at each strain level $(2 \%, 4 \%, 6 \%$, and $8 \%)$ for a perpendicular strain of $0 \%$ (blue) and 3\% (red). The transverse sample failed at a perpendicular strain of $3 \%$ and cycling strain of $8 \%$ so no data are shown. The longitudinal sample failed after a single cycle at a perpendicular strain of $3 \%$ and cycling strain of $8 \%$.

Figure 5. Plots of z-scores for sample orientation (A) and perpendicular strain (B) demonstrate that there is an effect of orientation but not perpendicular strain for most material property measures including elastic modulus (i), dissipation (ii), strain energy density (iii), initial stress (v), initial modulus (vi), and maximum stress (vii). Transition strain (iv) does not vary between longitudinal and transverse samples (A). A plot showing the interaction between orientation and 
perpendicular strain $(\mathrm{C})$ demonstrates that the effect of perpendicular strain is the same for longitudinal and transverse samples (i.e., there is no interaction between sample orientation and perpendicular strain).

Figure 6. Initial stress was greater in longitudinal (black) than transverse (gray) samples and increased from 0\% (solid) to 3\% (crosshatch) perpendicular strain (A). Initial modulus (B), maximum stress (C), strain energy density (D), hysteresis (E), and elastic modulus were greater in longitudinal versus transverse samples but did not change with perpendicular strain (see text for details).

Figure 7. The longitudinal layer of collagen fibers is on average, 2.4 times thicker than the transverse layer(s) of fibers (A). Histological sections of the fascia lata sectioned normal to the longitudinal (B) and transverse (C) planes show that collagen layer thickness is greater in the longitudinal (L) than transverse (T) orientation. Many samples, including the ones shown, had two layers of transverse collagen fibers. The diameter of the longitudinal collagen fibrils is on average, 39\% larger than the transverse fibrils (D). Scanning electron microscopy images demonstrate that the diameter of collagen fibrils is larger in the longitudinal (E) than transverse (F) layer. Scale bar is $80 \mu \mathrm{m}$ in (B) and (C) and $200 \mathrm{~nm}$ in (E) and (F). The asterisks in (A) and (D) demonstrate a significant difference between orientations ( $\mathrm{p}<0.05)$. 


\begin{tabular}{ll}
\multicolumn{1}{l}{ Abbreviations } & \\
FL & Fascia lata \\
SEM & Scanning electron microscopy \\
GB & Gluteobiceps \\
TFL & Tensor fascia lata \\
$\mathrm{L}_{0, \mathrm{C}}$ & Initial length in the cycling direction \\
$\mathrm{L}_{0, \mathrm{P}}$ & Initial length in the perpendicular direction \\
$\varepsilon_{\mathrm{P}}$ & Perpendicular strain \\
$\varepsilon_{\mathrm{C}}$ & Cycling strain \\
$\mathrm{PBS}$ & Phosphate-buffered saline
\end{tabular}


Click here to download high resolution image
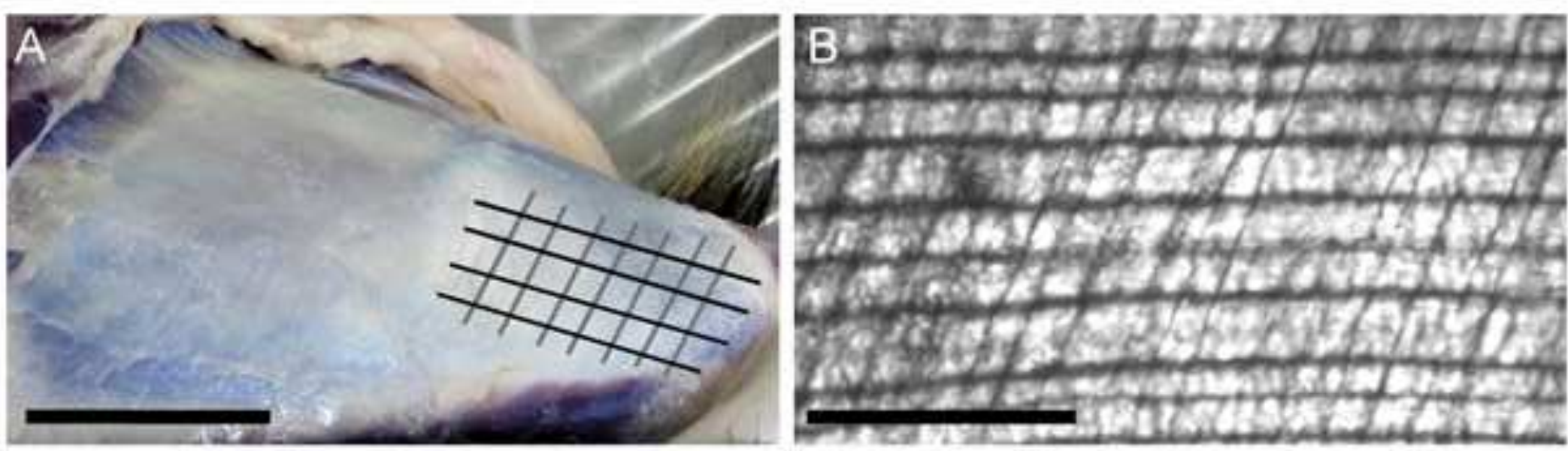
Click here to download high resolution image

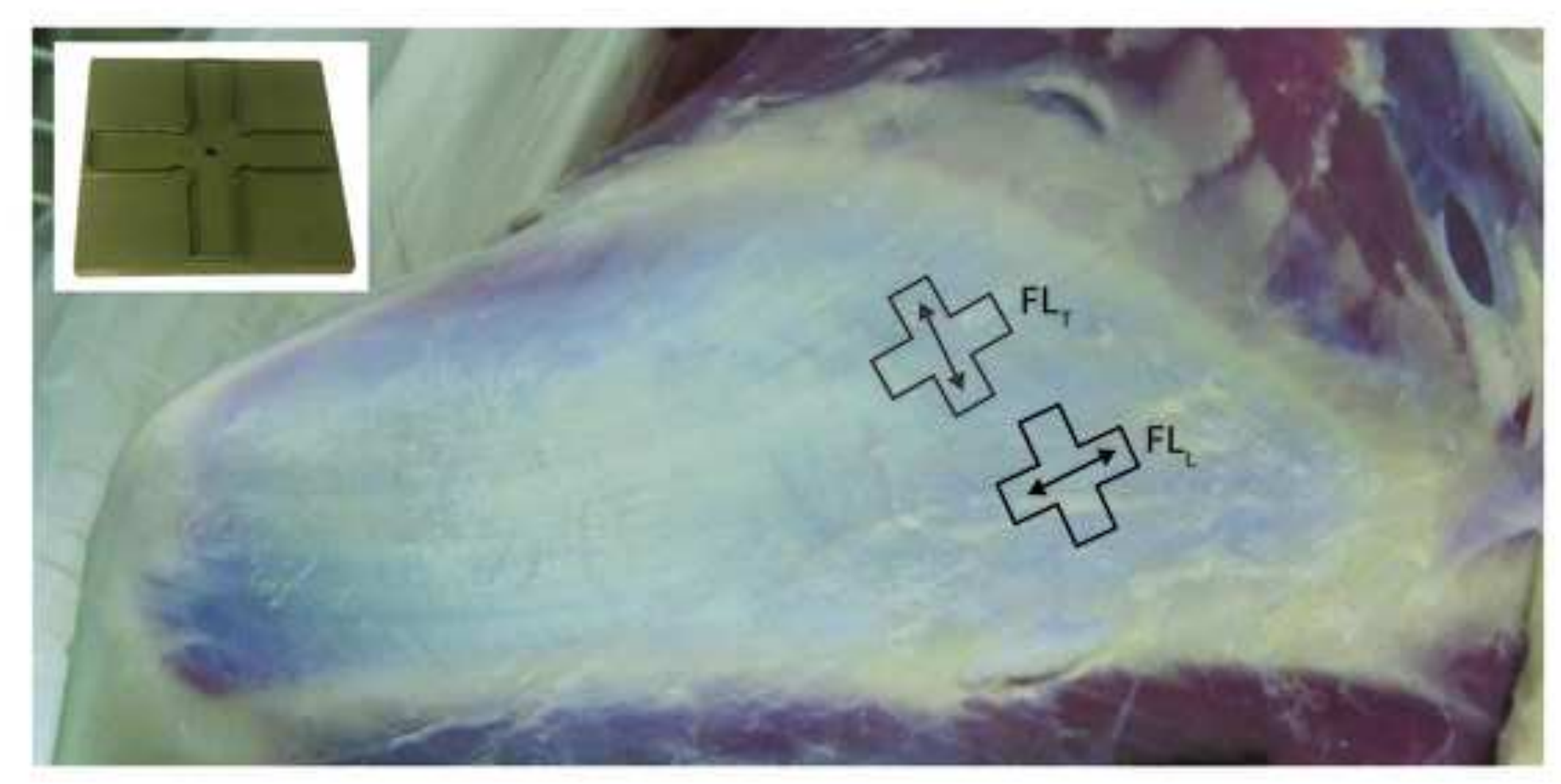

Figure 2
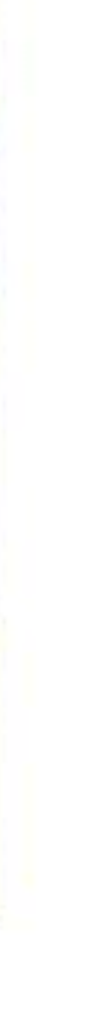
Click here to download high resolution image
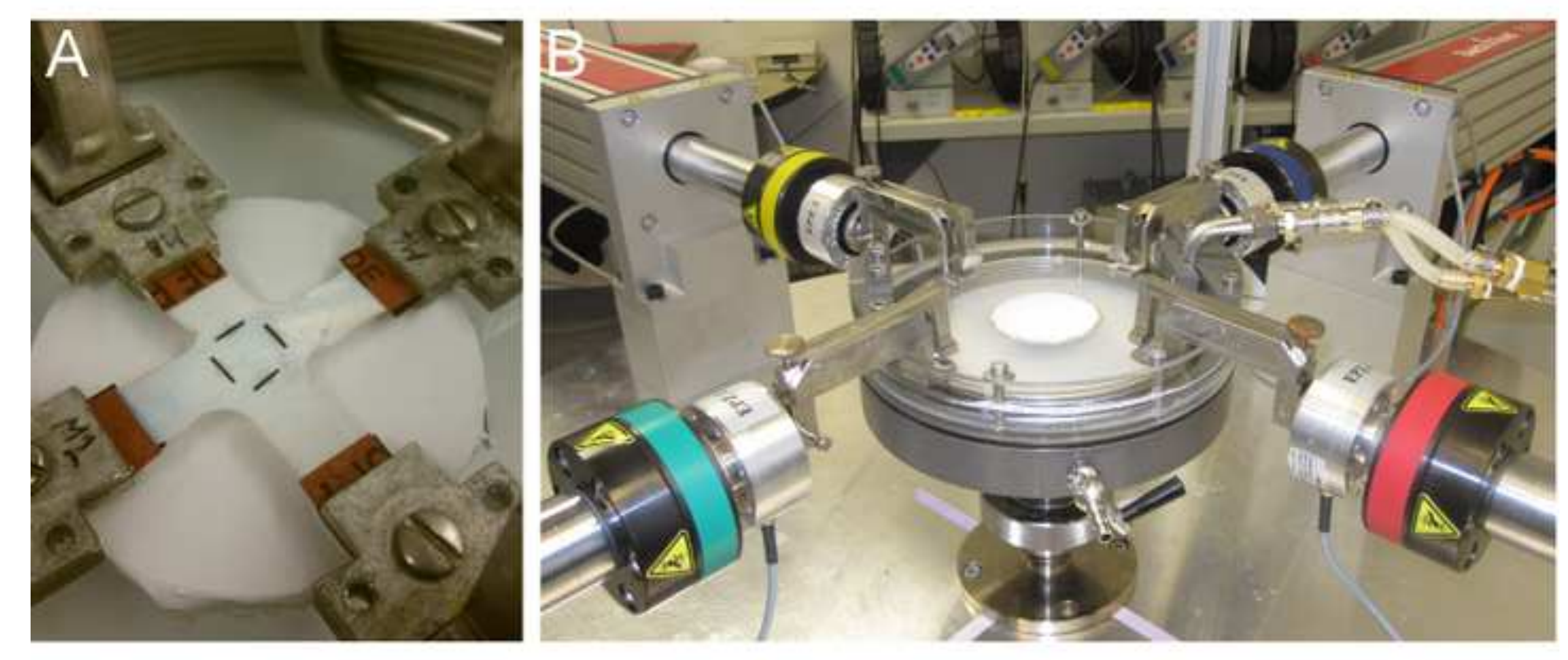

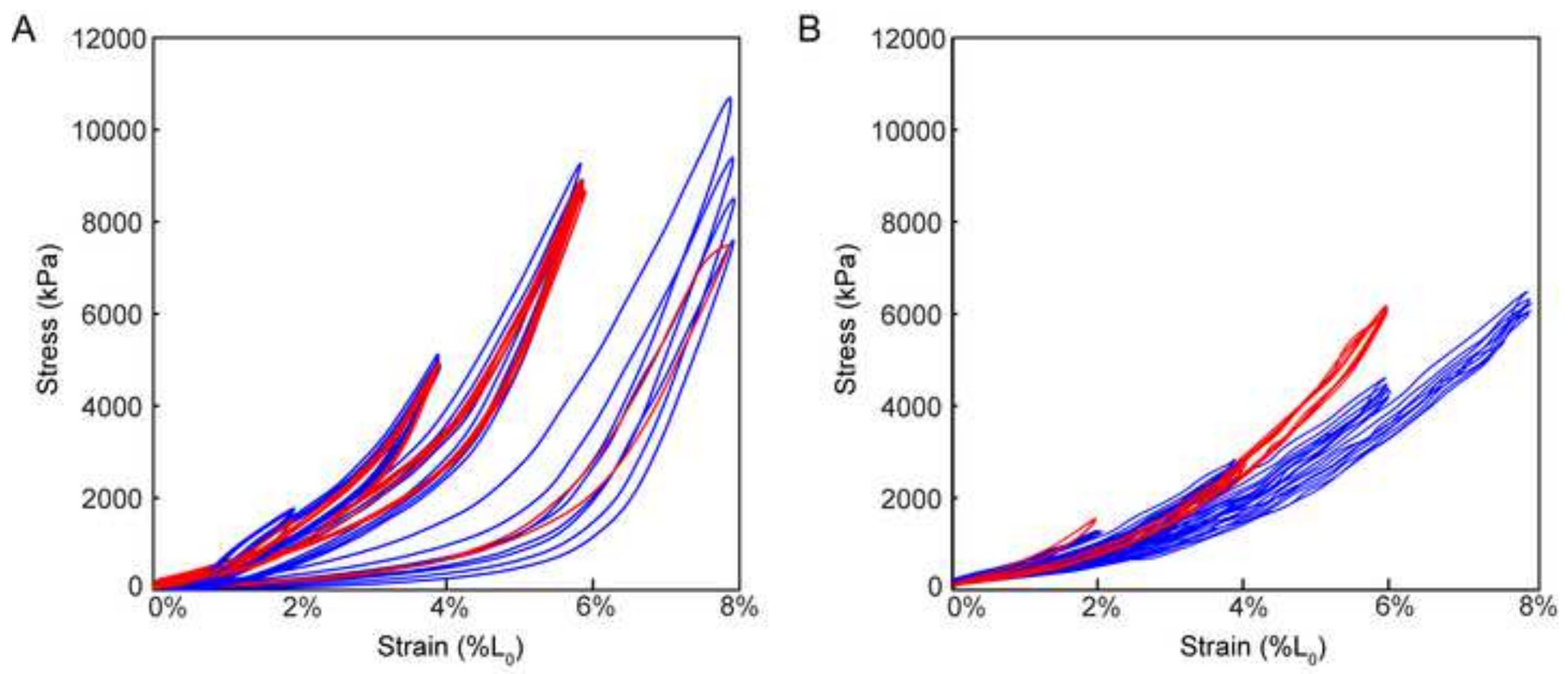

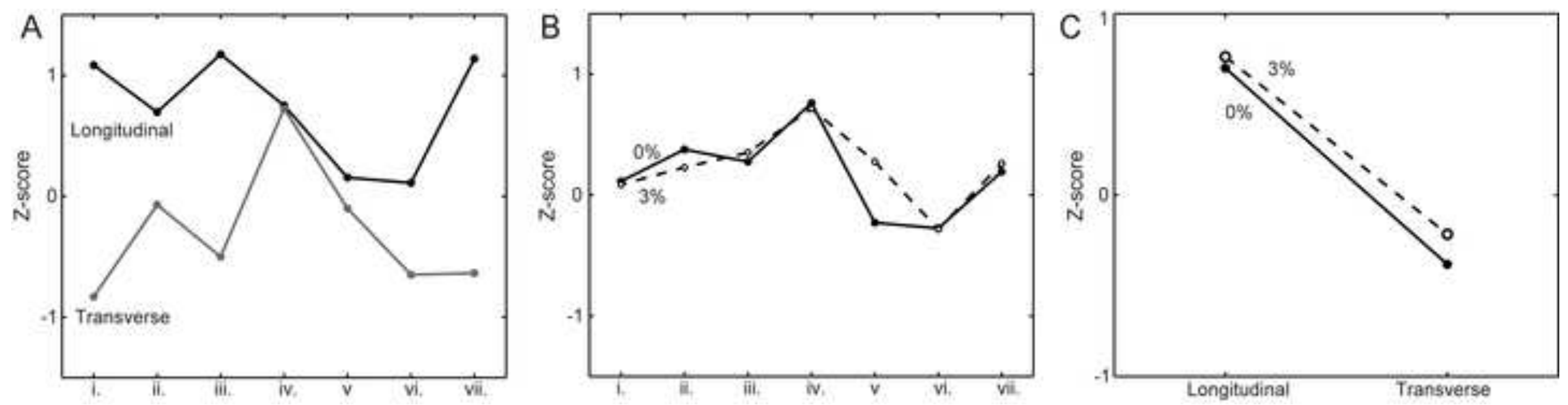


\section{Figure 6}

Click here to download high resolution image
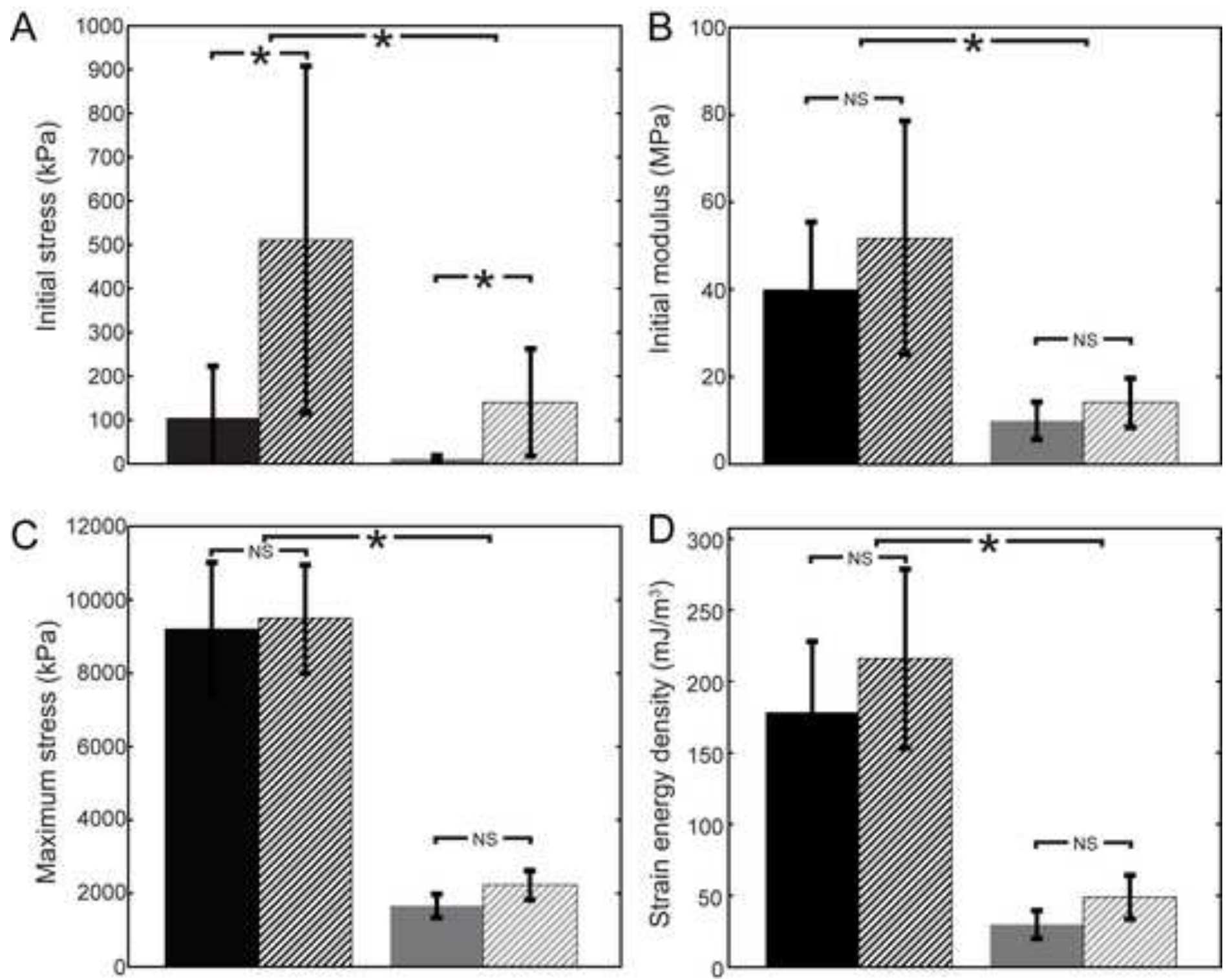

E
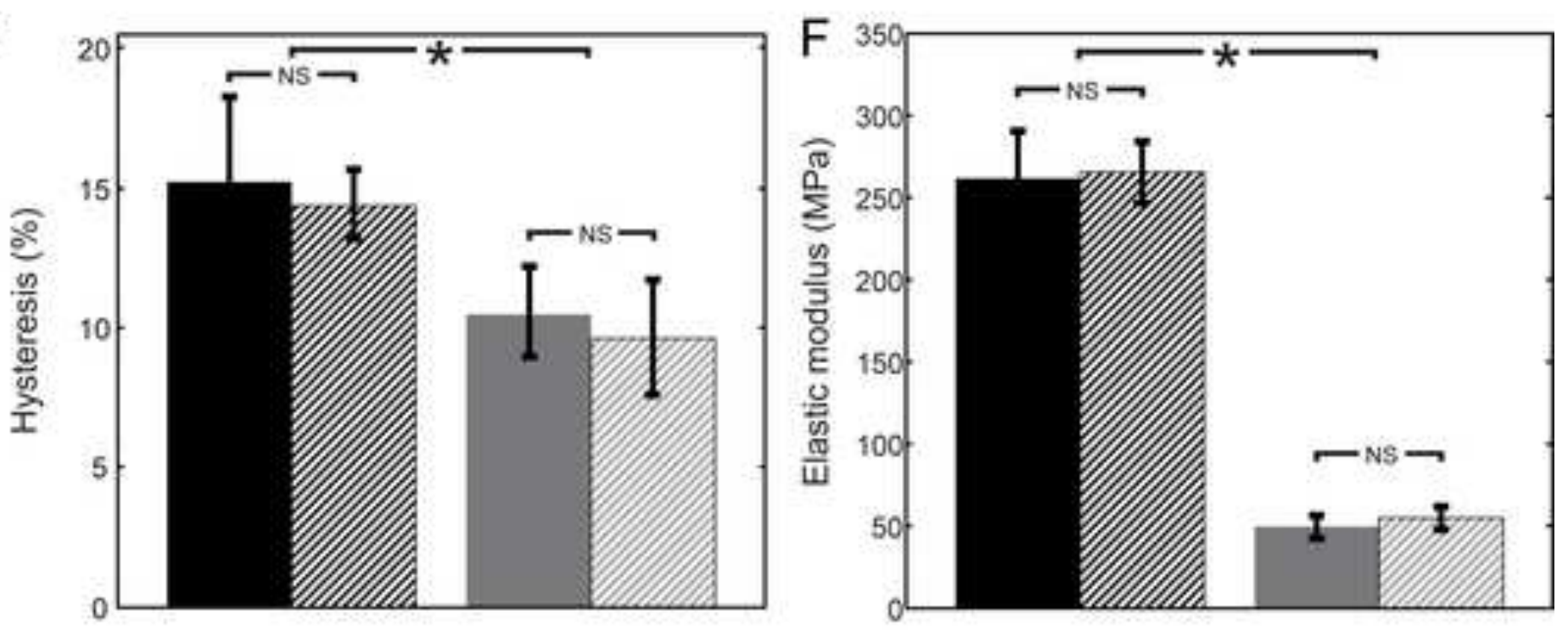
Table 1. Testing protocols consisted of a preconditioning sequence followed by cyclic strain testing sequences, in which perpendicular strain $\left(\varepsilon_{\mathrm{P}}\right)$ was initially set and held constant at $0 \%$ then $3 \%$ while cycling strain $\left(\varepsilon_{\mathrm{C}}\right)$ was cycled to each level.

\begin{tabular}{|c|c|c|c|c|}
\hline & Preload (N) & Testing sequence & Perpendicular strain $\left(\varepsilon_{P}\right)$ & Cycling strain $\left(\varepsilon_{C}\right)$ \\
\hline \multirow[t]{3}{*}{ Initial reference configuration set } & 0.08 & & & \\
\hline & & Preconditioning sequence & $0 \%$ & $2 \%$ \\
\hline & & & $3 \%$ & $2 \%$ \\
\hline \multirow[t]{9}{*}{ Reference configuration reset } & 0.08 & & & \\
\hline & & Testing sequence 1 & $0 \%$ & $2 \%$ \\
\hline & & & $3 \%$ & $2 \%$ \\
\hline & & Testing sequence 2 & $0 \%$ & $4 \%$ \\
\hline & & & $3 \%$ & $4 \%$ \\
\hline & & Testing sequence 3 & $0 \%$ & $6 \%$ \\
\hline & & & $3 \%$ & $6 \%$ \\
\hline & & Testing sequence 4 & $0 \%$ & $8 \%$ \\
\hline & & & $3 \%$ & $8 \%$ \\
\hline
\end{tabular}


Table 2. Goat fascia lata biaxial material properties.

\begin{tabular}{cccccccc}
\hline Sample & Elastic modulus & Hysteresis & Strain energy & Transition & Initial modulus & Initial & Maximum \\
orientation & $(\mathbf{M P a})$ & $(\boldsymbol{\%})$ & $\left(\mathbf{m J} / \mathbf{m}^{\mathbf{3}}\right)$ & strain $(\boldsymbol{\%})$ & $(\mathbf{M P a})$ & stress $(\mathbf{k P a})$ & $\mathbf{s t r e s s}(\mathbf{k P a})$ \\
\hline Longitudinal & $263.5 \pm 16.3$ & $14.8 \pm 1.6$ & $197.2 \pm 38.2$ & $3.3 \pm 0.3$ & $45.8 \pm 14.8$ & $307.7 \pm 206.5$ & $9357.1 \pm 1106.3$ \\
Transverse & $52.2 \pm 4.7$ & $10.1 \pm 1.3$ & $39.7 \pm 9.1$ & $3.1 \pm 0.3$ & $12.1 \pm 3.5$ & $75.6 \pm 61.6$ & $1947.5 \pm 254.0$ \\
\hline P-value & $<0.001$ & $<0.001$ & $<0.001$ & 1.00 & $<0.001$ & 0.034 & $<0.001$ \\
\hline
\end{tabular}

Data are expressed as mean \pm s.e.m.

Significant pair-wise comparisons are italicized $(\mathrm{p}<0.05)$. 
Table 3. Goat fascia lata biaxial material properties at $0 \%$ versus $3 \%$ perpendicular strain.

\begin{tabular}{|c|c|c|c|c|c|c|c|}
\hline \multirow[t]{2}{*}{ strain } & $\begin{array}{l}\text { Elastic } \\
\text { modulus }\end{array}$ & $\begin{array}{c}\text { Hysteresis } \\
(\%)\end{array}$ & $\begin{array}{l}\text { Strain energy } \\
\qquad\left(\mathbf{m J} / \mathbf{m}^{3}\right)\end{array}$ & $\begin{array}{l}\text { Transition } \\
\text { strain (\%) }\end{array}$ & $\begin{array}{c}\text { Initial } \\
\text { modulus }\end{array}$ & $\begin{array}{l}\text { Initial Stress } \\
\qquad(\mathbf{k P a})\end{array}$ & $\begin{array}{c}\text { Maximum stress } \\
(\mathbf{k P a})\end{array}$ \\
\hline & (MPa) & & & & (MPa) & & \\
\hline \multirow{2}{*}{ Longitudinal } & $261.6 \pm 28.9$ & $15.2 \pm 3.0$ & $178.4 \pm 49.7$ & $3.4 \pm 0.5$ & $39.7 \pm 15.7$ & $104.5 \pm 118.1$ & $9215.7 \pm 1835.3$ \\
\hline & $265.4 \pm 18.8$ & $14.4 \pm 1.3$ & $216.0 \pm 62.7$ & $3.2 \pm 0.5$ & $51.9 \pm 26.9$ & $511.0 \pm 396.5$ & $9498.5 \pm 1459.2$ \\
\hline \multirow{2}{*}{ Transverse } & $49.5 \pm 6.9$ & $10.5 \pm 1.7$ & $30.1 \pm 9.8$ & $3.0 \pm 0.4$ & $10.0 \pm 4.2$ & $11.2 \pm 7.2$ & $1661.0 \pm 318.5$ \\
\hline & $54.9 \pm 6.9$ & $9.7 \pm 2.2$ & $49.3 \pm 15.3$ & $3.3 \pm 0.4$ & $14.3 \pm 5.8$ & $140.0 \pm 122.3$ & $2234.1 \pm 384.4$ \\
\hline $\mathrm{P}$-value & 1.00 & 1.00 & 0.858 & 1.00 & 1.00 & $<0.001$ & 0.86 \\
\hline
\end{tabular}

Data are expressed as mean \pm s.e.m.

Significant pair-wise comparisons are italicized $(\mathrm{p}<0.05)$. 
Table 4. Structural properties of longitudinal and transverse collagen layers of goat fascia lata.

\begin{tabular}{lcc}
\hline & Collagen layer thickness $(\boldsymbol{\mu m})$ & Collagen fibril diameter $(\mathbf{n m})$ \\
\hline Longitudinal & $218.0 \pm 22.7$ & $127.0 \pm 9.2$ \\
Transverse & $94.7 \pm 6.5$ & $94.9 \pm 8.9$ \\
\hline P-value & 0.007 & 0.038 \\
\hline
\end{tabular}

Data are expressed as mean \pm s.e.m.

Significant pair-wise comparisons are italicized $(\mathrm{p}<0.05)$. 
Table 5. Material properties of goat fascia lata compared to other connective tissues.

\begin{tabular}{|c|c|c|c|}
\hline Tissue & Orientation & Elastic modulus (MPa) & Hysteresis (\%) \\
\hline \multirow[t]{2}{*}{ Goat fascia lata } & Longitudinal & $263.5 \pm 16.3$ & $14.8 \pm 1.6$ \\
\hline & Transverse & $52.2 \pm 4.7$ & $10.1 \pm 1.3$ \\
\hline Dog fascia lata $^{1}$ & Longitudinal & 390 & $11 \pm 2$ \\
\hline Human fascia lata $^{2}$ & & $397.5 \pm 17.1$ & \\
\hline \multirow[t]{2}{*}{ Turkey aponeurosis ${ }^{3}$} & Longitudinal & $744.4 \pm 51.6$ & $11.1 \pm 0.7$ \\
\hline & Transverse & $115.7 \pm 16.1$ & $15.7 \pm 0.9$ \\
\hline \multirow[t]{3}{*}{ Tendon } & -- & $305.5-612.8^{2}$ & \\
\hline & & $800-2000^{4}$ & $6-10$ \\
\hline & & $1270-1590^{5}$ & \\
\hline
\end{tabular}

Data are expressed as mean \pm s.e.m.

${ }^{1}$ Bennett et al. (1989)

${ }^{2}$ Butler et al. (1984)

${ }^{3}$ Azizi et al. (2009)

${ }^{4}$ Pollock and Shadwick (1994)

${ }^{5}$ Bennett et al. (1986) 
Form for Disclosure of Potential Conflicts of Interest
Click here to download Form for Disclosure of Potential Conflicts of Interest: COI_disclosure.pdf 